\title{
Rapid construction of genome map for large yellow croaker (Larimichthys crocea) by the whole-genome mapping in BioNano Genomics Irys system
}

\author{
Shijun Xiao ${ }^{1}$, Jiongtang $\mathrm{Li}^{2}$, Fengshou Ma ${ }^{3}$, Lujing Fang ${ }^{1}$, Shuangbin $\mathrm{Xu}^{1}$, Wei Chen ${ }^{1}$ and Zhi Yong Wang ${ }^{1 *}$
}

\begin{abstract}
Background: Large yellow croaker (Larimichthys crocea) is an important commercial fish in China and East-Asia. The annual product of the species from the aqua-farming industry is about 90 thousand tons. In spite of its economic importance, genetic studies of economic traits and genomic selections of the species are hindered by the lack of genomic resources. Specifically, a whole-genome physical map of large yellow croaker is still missing. The traditional BAC-based fingerprint method is extremely time- and labour-consuming. Here we report the first genome map construction using the high-throughput whole-genome mapping technique by nanochannel arrays in BioNano Genomics Irys system.
\end{abstract}

Results: For an optimal marker density of $\sim 10$ per $100 \mathrm{~kb}$, the nicking endonuclease Nt.BspQ1 was chosen for the genome map generation. 645,305 DNA molecules with a total length of $\sim 112 \mathrm{~Gb}$ were labelled and detected, covering more than 160X of the large yellow croaker genome. Employing IrysView package and signature patterns in raw DNA molecules, a whole-genome map of large yellow croaker was assembled into 686 maps with a total length of $727 \mathrm{Mb}$, which was consistent with the estimated genome size. The N50 length of the whole-genome map, including 126 maps, was up to $1.7 \mathrm{Mb}$. The excellent hybrid alignment with large yellow croaker draft genome validated the consensus genome map assembly and highlighted a promising application of whole-genome mapping on draft genome sequence super-scaffolding. The genome map data of large yellow croaker are accessible on lycgenomics.jmu.edu.cn/pm.

Conclusion: Using the state-of-the-art whole-genome mapping technique in Irys system, the first whole-genome map for large yellow croaker has been constructed and thus highly facilitates the ongoing genomic and evolutionary studies for the species. To our knowledge, this is the first public report on genome map construction by the whole-genome mapping for aquatic-organisms. Our study demonstrates a promising application of the whole-genome mapping on genome maps construction for other non-model organisms in a fast and reliable manner.

Keywords: Large yellow croaker, Gene map, Whole-genome mapping, Genome assembly

\section{Background}

Large yellow croaker (Larimichthys crocea), belonging to the family of Osteichthyes and the order of Perciformes, is an economic fish in China and East Asia [1]. With an annual product of 90 thousand ton, the species is one of the most important mariculture fish in China [2]. Nowadays, the direct annual marker value of large yellow

\footnotetext{
* Correspondence: zywang@jmu.edu.cn

'Key Laboratory of Healthy Mariculture in the East China Sea, Ministry of Agriculture; Fisheries College, Jimei University, Yindou Road, Xiamen, P.R. China Full list of author information is available at the end of the article
}

croaker is about 60 thousand dollar. In the last decades, the wild population of the species has collapsed due to over-fishing and habitat degeneration [3, 4]. Both the environmental changes and over-dense aquaculture pose enormous challenges on economic trait degeneration, stress adaption and susceptibility to infectious disease for large yellow croaker [1]. Therefore, it is an urgent requisite to explore the association analysis of functional genes with aquaculture performance in order to breed new large yellow croaker strain with better economic 
traits and higher anti-infection to meet the requirement of the modern aquaculture industry [2]. Many literatures have been published for gene functions [5, 6], mitochondrial genome [7] and transcriptome analysis [8] for large yellow croaker in the last few years. Genetic maps of the large yellow croaker has also been reported using amplified fragment length polymorphism (AFLP) and microsatellites markers aiming at pseudo-chromosome construction and functional gene identification $[9,10]$. Recently, scientists have reported the draft genomes of large yellow croaker in 2014 and 2015 (referred as 2014draft and 2015draft hereafter, respectively) [11, 12], which highly facilitate the functional gene mapping and genome-wide association studies (GWAS). In spite of these efforts in genomic analysis of large yellow croaker, a physical map for the species is still lacking, which hinders the fine genomic sequence assembly and further investigation of complex traits by multi-omics analysis.

The advent and development of Next Generation Sequencing (NGS) have already brought scientific studies in many fields including agriculture and medicine into an era of genomics in the last decade $[13,14]$. Nowadays, it is possible to de novo assembly a draft genome of a species in several months [15]. However, the genome assembly from short NGS reads remains a challenge [16], especially for aquatic organisms with high repeat context and complex genomic heterozygosity. Physical mapping is one prevalent technique that used to facilitate sequence assembly and functional gene location for genomic projects of many species [17-19]. It employs specific fingerprints along DNA molecules and, more importantly, can span large repeat and heterozygous regions; thus the signature patterns in physical maps provide valuable framework for fine genomic sequence assembly. Traditional BAC-based physical mapping technologies have been used in many aquatic fish species, including Nile tilapia (Oreochromis niloticus) [20], Atlantic salmon(Salmo salar) [21] channel catfish (Ictalurus punctatus) [22], Asian sea bass (Lates calcarifer) [23], rainbow trout (Oncorhynchus mykiss) [24] and half-smooth tongue sole (Cynoglossus semilaevis) [25]. Yet, BAC-based fingerprint experiments are extremely time consuming and labour costly. In the last few years, scientists have invented highthroughput methods to identify restraint sites of megasized DNA molecules directly, leading to mainly two novel physical mapping technologies: optical mapping [26] and whole-genome mapping [27-29]. In optical mapping, large DNA molecules are fixed and digested on polylysine-treated glass surfaces and DNA fragments and digestions are visualized by fluorescence microscopy, so that ordered restriction maps of those DNA molecules are derived from digital images [26, 30-32]. The advent of optical mapping has led to many successful applications in micro-organism chromosome map constructions [31-33]. However, the non-linear fixation of DNA molecules on glass surfaces and completed sequence digestion influence native molecular stressed conformations and pose tremendous challenges for the accurate estimation of signature length in DNA fragments. To overcome those problems, scientists have recently invented the whole-genome mapping by utilizing nanochannel arrays and nicking endonuclease to minimize the bias for the nicking site detection [34, 35]. Equipped with this state-of-the-art technology, BioNano Genomics Irys system is developed and could linearize a single DNA molecule in one $45 \times 45 \mathrm{~nm}$ nanochannel with buffer to detect DNA molecule length and locations of nicking sites [34]. In the last few years, Irys system has been used to build genome maps as well as to guide high-confidence assembly of extra-ordinary complex genomic regions in aegilops tauschii [36] and in human [37]. Given such advantages, the genome map application of the wholegenome mapping in Irys system for non-model organisms is rather promising; however, to our best knowledge, a public report of genome map construction applying Irys system for non-model organisms has not been published so far.

Here in this study, we employed Irys system to construct a whole-genome map of large yellow croaker. A large amount $(\sim 120 \mathrm{~Gb})$ of DNA fragment information were detected from the system. The high coverage $(\sim 160 X)$ of raw data assured sufficient DNA molecules for genome map assembly. The genome maps were validated by the alignment with draft genome sequences. We believe the constructed whole-genome map for large yellow croaker can provide a valuable framework for super-scaffolding draft genome sequences, building pseudo-chromosomes and facilitating the following integration analysis with genetic maps.

\section{Results and discussions}

\section{Nicking endonuclease selection}

Four candidate enzymes in Table 1, Nt.BspQ1, Nb.Bsml, $\mathrm{Nb} . \mathrm{BbvCI}$ and $\mathrm{Nb} . \mathrm{BsrD} 1$, were provided for the wholegenome mapping in Irys system. To select a restriction endonuclease enzyme with a proper label density for the

Table 1 Label density estimation. Label density estimation for four restriction endonuclease enzyme candidates

\begin{tabular}{lcc}
\hline Enzyme & $\begin{array}{l}\text { Nick density } \\
\text { (nicks per 100 kb) }\end{array}$ & $\begin{array}{l}\text { Label density } \\
\text { (labels per 100 kb) }\end{array}$ \\
\hline Nt.BspQ1 & 13.7 & 11.0 \\
Nb.Bsml & 35.9 & 22.6 \\
Nb.BbvCl & 17.6 & 13.4 \\
Nb.BsrD1 & 41.1 & 24.4 \\
\hline
\end{tabular}


whole-genome mapping, the public draft genome 2014draft [11] of large yellow croaker was used to estimate the endonuclease site density. As shown in Table 1, Nb.BsrD1 and Nt.BspQ1 exhibited the highest (41.1) and lowest (13.7) site density, respectively. Based on previously estimated resolution for the platform, the experimental label densities were evaluated in silico by omitting nicking sites within 500 bp to each other; therefore the label densities were smaller than nick site densities because of the limited resolution between nick sites. To satisfy the optimal label density of $\sim 10$ per $100 \mathrm{~kb}$ in Irys system, we selected the nicking endonuclease of Nt.BspQ1 with the specific motif of GCTCTTC for the whole-genome mapping (Table 1). According to the empirical performances in other species, the whole genome average label density may fluctuate by $15 \%$ around the pilot estimation, suggesting a reasonable average label density of Nt.BspQ1 nicking sites for large yellow croaker genome should fall into the range of $9.35 \sim 12.85$ per $100 \mathrm{~kb}$.

\section{DNA molecules and labels}

DNA lengths and nicking sites information were recorded as digital images in Irys system (see detail in Material and Method). From recognition processes of the resulted digital images, we obtained 645,305 valid DNA molecules longer than $100 \mathrm{~kb}$. The raw DNA molecules number and length distribution were depicted in Fig. 1a. The N50 length of all DNA fragments was $\sim 176 \mathrm{~kb}$. Notably, the longest DNA molecule was $2.88 \mathrm{Mb}$, which was even comparable to the longest assembled physical map in previous literatures. Megasized DNA molecules resulted from long DNA library construction and nanochannel design [28] in Irys system would extremely improve the genome map assembly. The total throughput of raw DNA molecules was about $112 \mathrm{~Gb}$, covering about 160X of large yellow croaker genome. The high-throughput of raw DNA molecules suggested that Irys system outperformed previous reported BAC-based fingerprint method on raw data output $[19-25,38]$ and provided sufficient DNA fragments for a reliable whole-genome map assembly. We noted that almost half of DNA lengths were catalogued as 100 $\sim 150 \mathrm{~kb}$ but those molecules only account for $35 \%$ $(\sim 40 \mathrm{~Gb})$ of the overall throughput, implying longer DNA molecules contribute significantly to the total data. A plenty of DNA molecules $(5,758)$ were longer than $500 \mathrm{~kb}$ and the total length of those molecules was $\sim 4.1$ $\mathrm{Gb}$, covering $5.5 \mathrm{X}$ of large yellow croaker genome (Fig. 1a). We listed the detailed DNA molecule numbers and average depths information against various length thresholds in Additional file 1: Table S1. The average depth was still larger than 100X even with a length threshold as long as $150 \mathrm{~kb}$.
To validate the label density within DNA molecules, we illuminated the average label density against DNA length in Fig. 1b. The label density of Nt.BspQ1 was rather stable as $\sim 10.6$ in all length categories, which excellently agreed with our previous pilot theoretical evaluation. However, we found that the label densities in one DNA molecule varied from 0.08 to 46.4, implying the heterogeneous nicking site distribution along large yellow croaker genome. To evaluate signature patterns of raw DNA molecules and to estimate accuracy of motif labelling by Irys system, we aligned those signature patterns in DNA molecules longer than $150 \mathrm{~kb}$ to the draft genomes of large yellow croaker (see Material and Method for detail). As a result, $\sim 60$ and $\sim 67 \%$ of raw DNA molecules were successfully thoroughly mapped upon $2014 \mathrm{draft}$ and $2015 \mathrm{draft}$ genome sequences, respectively. The alignment ratio was reasonable for three main reasons: firstly, the draft genome sequences were not completed. Because of length scale of DNA molecules $(>100 \mathrm{~kb})$, the incompleteness of draft genome would influence the raw data mapping, especially for those DNA molecules locating at sequence ends or in unassembled genome sequences; secondly, the wholegenome mapping and de novo sequencing experiments were carried out with different individuals, therefore inherent genetic diversifications unavoidably raised the divergence for alignments; thirdly, false positives (false present markers in DNA molecules) and false negatives (true markers absent in DNA molecules) caused by enzymatic activities, limited labelling resolution, camera recording and software processing also mislead DNA mapping. Our analysis also implied that the bioinformatics utilities (IrysView packages) had much room for further improvement for more efficient genome map applications. Based on mismatches observed in alignments between raw DNA molecules and genome sequences, we estimated the average false positive to genome sequences in this experiment to be 1.5 per $100 \mathrm{~kb}$, which was close to the estimation of 1 per $100 \mathrm{~kb}$ in the whole-genome mapping for $E$. coli. Note that inherent sample-wise genetic diversifications of individuals may also contribute to the false positive rate estimation; thereby the technical false positive rate should be lower. Meanwhile, we calculated the average Bases per pixel (BPP) in the labelling to be $504 \mathrm{bp}$, implying the technique exhibited an excellent resolution to detect fluorescence labels $\sim 500$ bp around real nicking sites in the experiment. Based on above analyses, we concluded that the wholegenome mapping in Irys system provided high throughput unbiased raw DNA molecules with a proper resolution for large yellow croaker genome map construction.

\section{Whole-genome map assembly from DNA molecules}

To build a whole-genome map, raw DNA molecules longer than $100 \mathrm{~kb}$ were used in Assembler utility of IrysView 
(A)

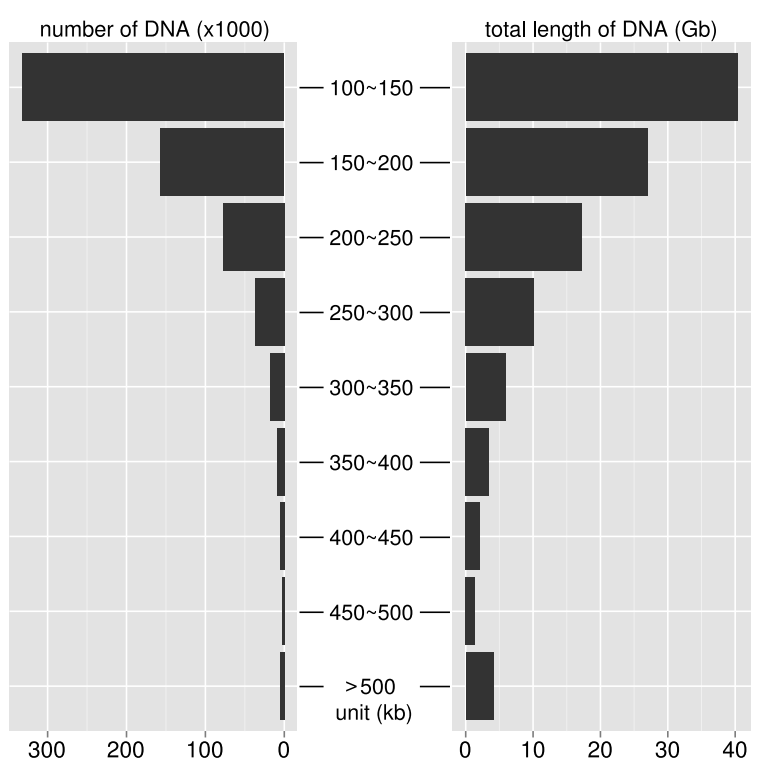

(B)

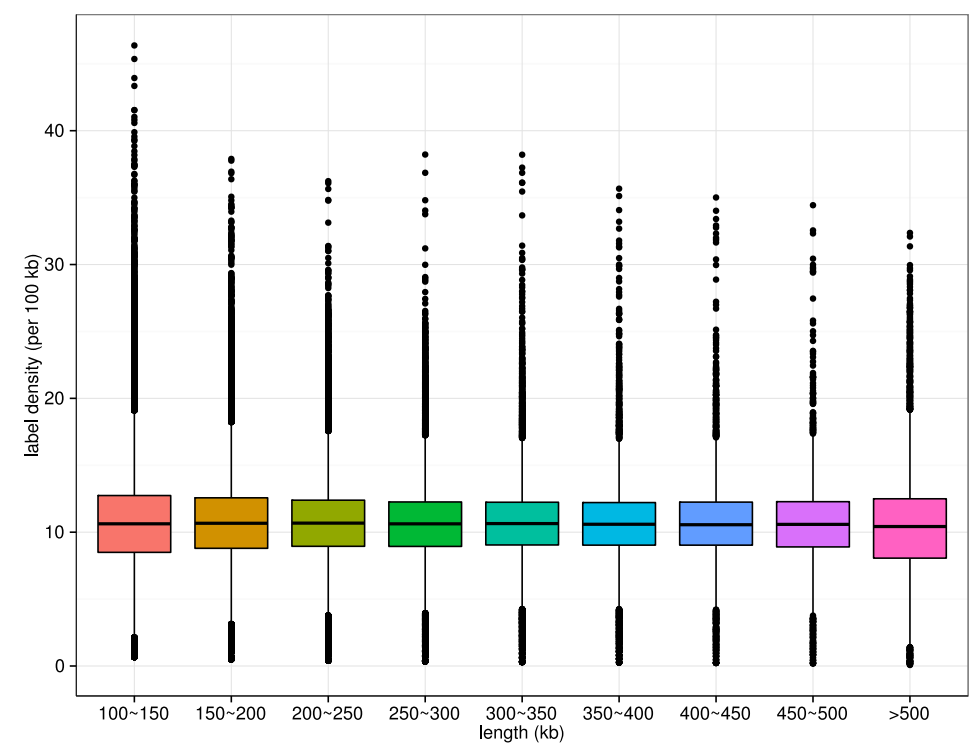

Fig. 1 The number, total length and label density distribution of raw DNA molecules from whole-genomic mapping. a Sequence number (left) and total length (right) of raw DNA molecules for each length interval; b Label density in boxplot for each length interval. The estimated overall average label density was $\sim 10.6$

package to construct consensus genome maps of large yellow croaker. As a result, 686 genome maps were assembled with a total length of $727 \mathrm{Mb}$, which was excellently consistent with the genome size estimation of $725 \pm 14.6 \mathrm{Mb}$ in flow cytometry experiments [39]. Additional file 1: Figure S1 illuminated an example of consensus map construction from raw DNA molecules. As shown in Table 2, the N50 length of the assembled genome maps for large yellow croaker genome was $\sim 1.7 \mathrm{Mb}$, with the longest and smallest map to be $105 \mathrm{~kb}$ and $9 \mathrm{Mb}$, respectively. The average length of genome maps was $\sim 1.06 \mathrm{Mb}$ (Fig. 2). We observed that the N50 length was significantly longer than the mean length of genome maps, implying a quality whole-genome map assembly. Among all genome maps, 94 of them $(\sim 14 \%)$ were longer than $2 \mathrm{Mb}$, representing $305 \mathrm{Mb}(\sim 2 \%)$ of the whole large yellow croaker genome.

To investigate how DNA molecule depth influenced genome map assembly and to determine the minimal 
Table 2 Genome map and draft genome sequence statistics. Basis statistics for raw DNA molecules, assembled genome maps and published draft genome sequences

\begin{tabular}{lllll}
\hline & Raw DNA molecules & Genome maps & 2014draft [11] & 2015draft [12] \\
\hline Number & 645,305 & 686 & 3,478 & 67013 \\
Total length $(\mathrm{Mb})$ & 111,830 & 727 & 644 & 1,030 \\
N50 $(\mathrm{kb})$ & 176 & 1,714 & 498 & 0.5 \\
$\min (\mathrm{kb})$ & 100 & 105 & 2.0 & 4,914 \\
$\max (\mathrm{kb})$ & 2,880 & 9,002 & 3,825 & \\
\hline
\end{tabular}

data required for a proper whole-genome mapping of large yellow croaker, subsets of DNA fragments with various depths were sampled from total raw DNA molecules. Figure 3 showed that N50 length of genome maps exceeded $1 \mathrm{Mb}$ with a molecule depth of $50 \mathrm{X}$ and increased almost linearly by improving molecule depth from $30 \mathrm{X}$ to $70 \mathrm{X}$, while the corresponding total map number decreased sharply from 1162 (30X) to 799 (70X). Although a slightly weakening tendency was observed with molecule depth from $70 \mathrm{X}$ to 100X, both map number (715) and N50 length $(\sim 1.58 \mathrm{Mb})$ were significantly improved. We found that the map number and N50 length under the depth of 100X have already surpassed draft genome sequences of 2014 draft and 2015 draft for large yellow croaker (Table 2). The further accumulation of molecule depth larger than 100X led to relatively smaller changes of map number and N50 length. Considering the trade-off between experimental cost and proper genome map assembly, a molecule depth of $\sim 100 X$ may represent an optimal molecule depth with the best cost/performance ratio for the whole-genome mapping of large yellow croaker. This analysis offered a valuable reference for the following projects using the whole-genome mapping technique to construct genome maps for other non-model organisms, but the caution should always be taken that the optimal molecule depth might vary with genomic complexity in organisms.

\section{Genome map evaluation}

Contrast with previous reports based on BAC-end fingerprint, the whole-genome map in this work was

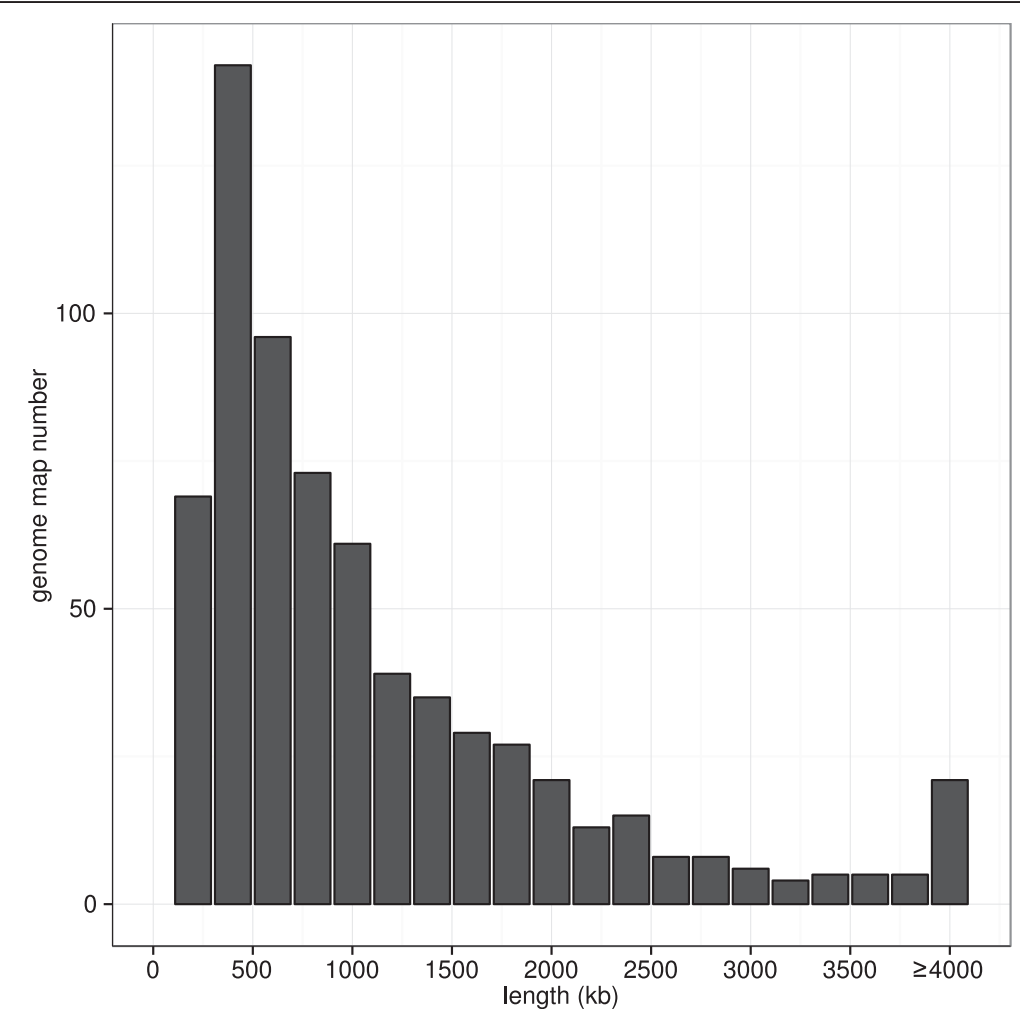

Fig. 2 The length distribution of genome maps for large yellow croaker. Note that all maps longer than $4 \mathrm{Mb}$ were accumulated in the last bar 


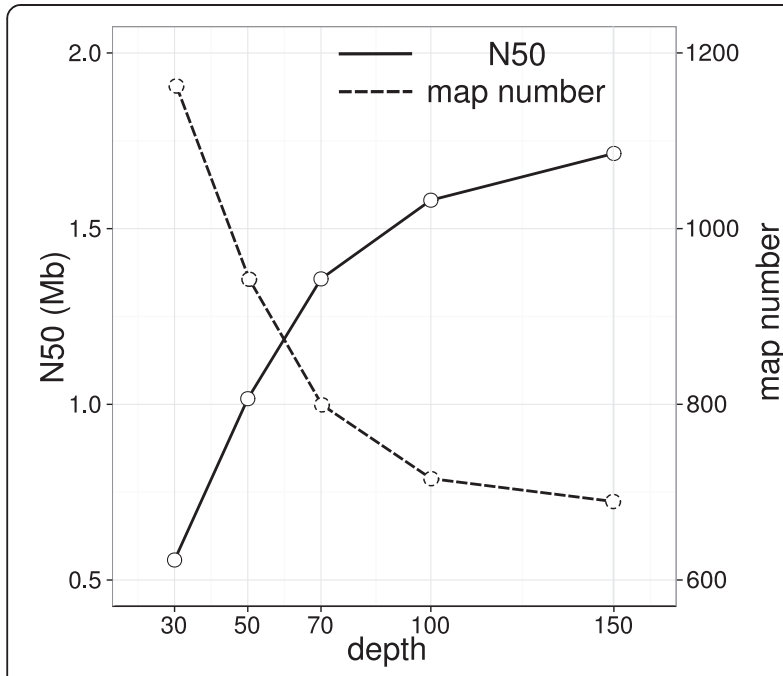

Fig. 3 N50 and map number of the whole-genome map assembly under different molecule depth. We randomly sampled subsets from total raw DNA molecules to assembly genome map, aiming to estimate how molecule depth influenced N50 length (solid line; left Y-axis) and map number (dashed line; right $Y$-axis). All parameters were identical for all map assembly

constructed directly from mega-sized genomic DNA molecules; therefore it was difficult to evaluate genome maps via PCR amplifications by primers designed from BAC clones. However, the length scale of consensus maps enables us to directly compare all published genome sequences with the whole-genome map. The underlying philosophy was that if genome maps were correctly assembled, there should be a large proportion of alignments between genome sequences and maps. We believe that the hybrid alignment of genome sequences and map is extremely essential because the analysis not only evaluates the accuracy of genome maps but also answer the question that to what extent can the whole-genome map facilitate the genome sequence super-scaffolding.

Since the published genome sequences of large yellow croaker were remarkably more fragmented than the assembled genome maps (Table 2), we aligned all genome sequences to the whole-genome map by RefAligner utility in IrysView package (see detail in Materials and methods). To improve the accuracy of the hybrid alignment, only genome sequences with more than 6 nicking sites, leaving 1,870 fragments, were used in the analysis. As shown in Fig. 4, we found that 1,154 ( 62\%) and 931 ( 50 \%) genomic sequences aligned upon genome maps with a mapping length ratio higher than 0.7 and 0.8 for 2014draft, respectively. More than 300 genome sequences were thoroughly aligned to genome maps (mapping length ratio $\geq 0.95$ ). The excellent alignments were also observed for genome sequences from 2015draft (Fig. 4). Considering the fact that genome sequences of large yellow croaker were also fragmented, our alignment results confirmed the reliability of the whole-genome map constructed from raw DNA molecules.

To visualize nicking site alignments between genome sequences and maps, Fig. 5 illuminated the detail schema of local alignment of 2014draft genome sequence 41 (G41) upon genome map 93 (M93). The excellent accordance of nicking sites spanning $\sim 1 \mathrm{Mb}$ region confirmed the accuracy of the whole-genome mapping. More notably, we found that many genome maps were thoroughly aligned by shorter reference genome sequences. Examples were demonstrated in Fig. 6a, b that genome map M129 and M4 were covered by two genome sequence groups of G149, G347, G216, G448 and G70 from 2014draft and G1478, G1866, G1180, G1396, G2035 and G1853 from 2015draft, respectively. The gaps between adjacent alignment boundaries likely represent regions that failed to cover in genomic NGS sequencing projects or low-complexity sequences. In addition, the mapping directions were also observed in those hybrid alignments; for example 2014draft G448 was found to reversely aligned to M129 (Fig. 6a). Given the multi-alignment information on genome maps, orders of genome sequences along chromosomes, as well as their directions, were clearly assigned. The alignment analysis of genome sequences upon the whole-genome map demonstrated that genome maps successfully strode gapped regions in genomic NGS sequencing experiments and thus served as an ideal guidance for the following genome sequences super-scaffolding and integrative analysis with genetic maps.

\section{Conclusions}

Large yellow croaker is an important commercial fish in China and East Asia, however, genomic resources of the species, especially for physical maps, are still lacking, hindering the following genomic information integration and functional gene mapping. The construction of wholegenome physical map could facilitate genome sequence assembly and map-based gene identification [25, 40]. Therefore, a completed and accurate physical map of large yellow croaker could provide valuable reference for genomic studies of this important species.

The traditional physical map construction method based on BAC libraries and restriction fingerprinting is extremely time- and labour-expensive. More importantly, those libraries barely cover $100 \%$ of the genome, because there would be some missing genomic regions caused by restriction enzyme bias, leaving gaps in the assembled physical map [25]. Employing nano- and optical-technologies, Irys system enables researchers to perform the whole-genome mapping in a highthroughput manner by nicking endonuclease in megasized DNA molecules directly [27]. The system has been applied in high-resolution genome map construction 


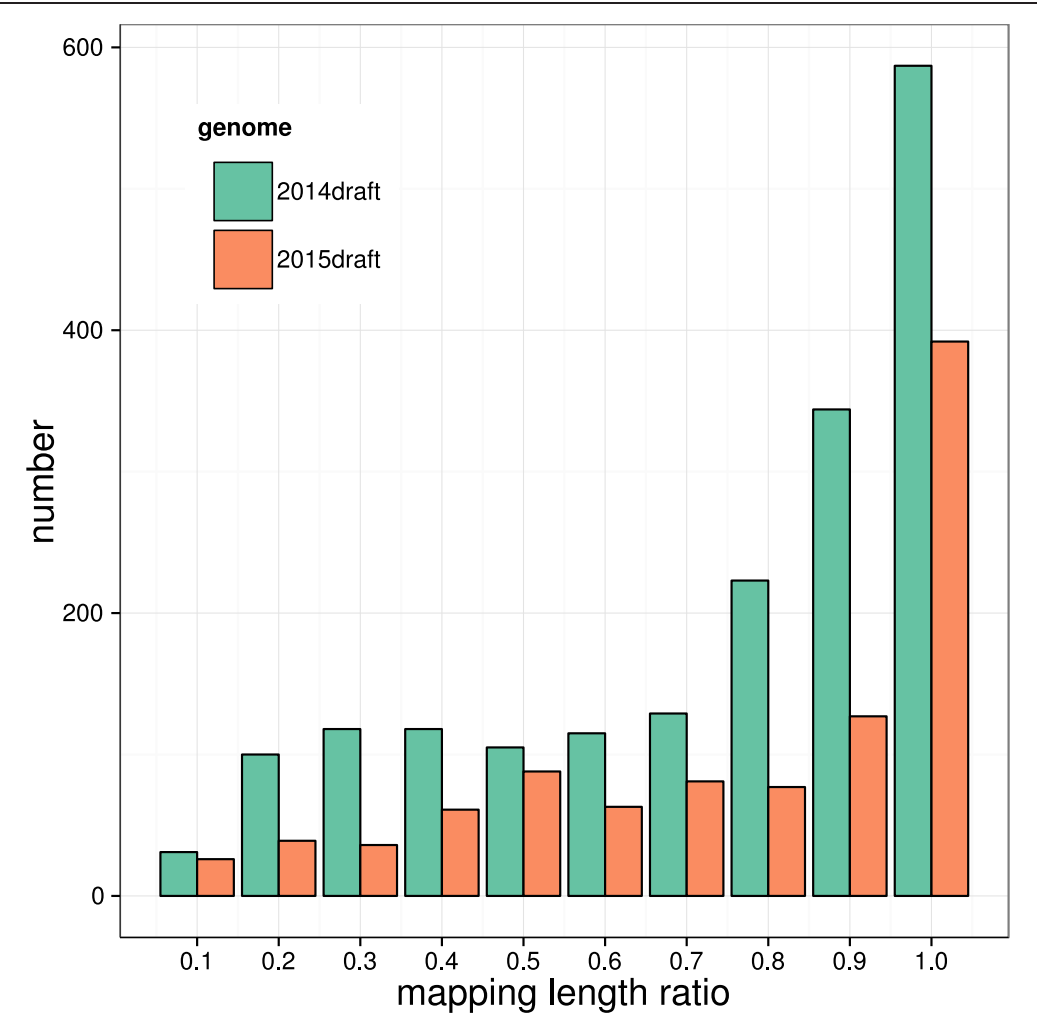

Fig. 4 Mapping length ratio distribution for the alignment of genome sequences upon genome maps. The mapping length ratio, defined as map length divided by total length, were estimated for reference genome 2014draft [11] (green) and 2015draft [12] (orange). Only mappings with the highest confidence score of each genomic sequence were collected for the analysis. Note that small mapping length ratios may represent large scale nicking site heterozygosity or mappings at map ends

for complex regions of several model species, such as human and wheat [34-36]. In this work, we employed the state-of-the-art technique to probe signature patterns in mega-sized DNA molecules and assembled the first whole-genome map of large yellow croaker. With GCTCTTC motif recognized by the nicking endonuclease of Nt.BspQ1, signature patterns in 645,305 DNA molecules $(\geq 100 \mathrm{~kb})$ were detected from the system and assembled into a whole-genome map of large yellow croaker, resulting into 686 maps with a N50 length of $\sim 1.7 \mathrm{Mb}$. The total length of genome maps was $727 \mathrm{Mb}$, agreeing well with the flow cytometry estimation [39]. The influence of DNA molecule depth on map assembly were investigated and discussed. The hybrid alignment between constructed genome maps and draft genome sequences validated the map assembly and illuminated the potential application of the whole-genome mapping for draft genome sequences super-scaffolding.

With the completed and reliable map resources, the comprehensive integration analysis of genetic map and genome map could highly improve the genomic sequence assembly, evolutionary investigations and genomic selections of large yellow croaker. Meanwhile, this is the first public report on genome map construction for none-model organisms by Irys system. Our studies have validated the whole-genome mapping technique and provided valuable reference for rapid genome map construction of other organisms.

\section{Methods}

\section{Ethics statement}

This study was approved by the Animal Care and Use committee of Fisheries College of Jimei University.

\section{Sample preparation and mega-sized DNA extraction}

Large yellow croaker samples were obtained from the breeding base of Jimei University in Ningde, Fujian, China. Venous blood samples from fish tails were collected for genome DNA extraction. To extract long genomic DNA molecules from blood samples, peripheral mononuclear cells (PBMCs) from whole blood were isolated after density gradient centrifugation with Ficoll-Paque PLUS medium and BioNano's commercial plug lysis protocol. The megabase-sized DNA molecules were then extracted according to IrysPrep TM Plug Lysis Long DNA Isolation protocol. Qubit 2.0 was used to quantify DNA concentration in solution. DNA samples met the following criterions were used 


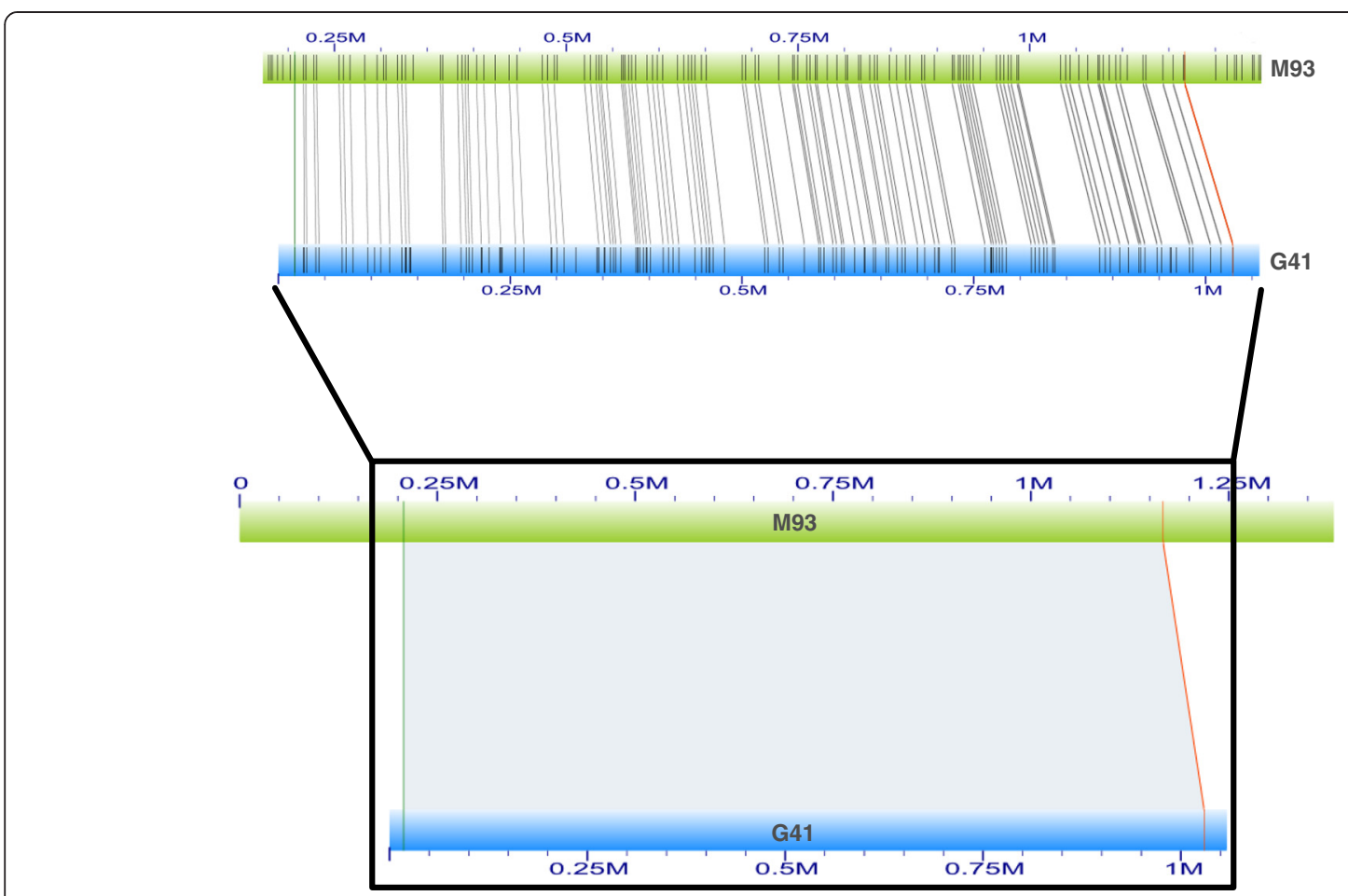

Fig. 5 The detail nicking site alignment of genome sequences to genome maps. The genome sequence and genome map were shown in light blue and green colour and the corresponding names begun with $\mathrm{G}$ and $\mathrm{M}$, respectively. The zoom in inset illuminated the detail nicking site alignments. The light green and red boundaries represented the beginning and ending sites for the alignment

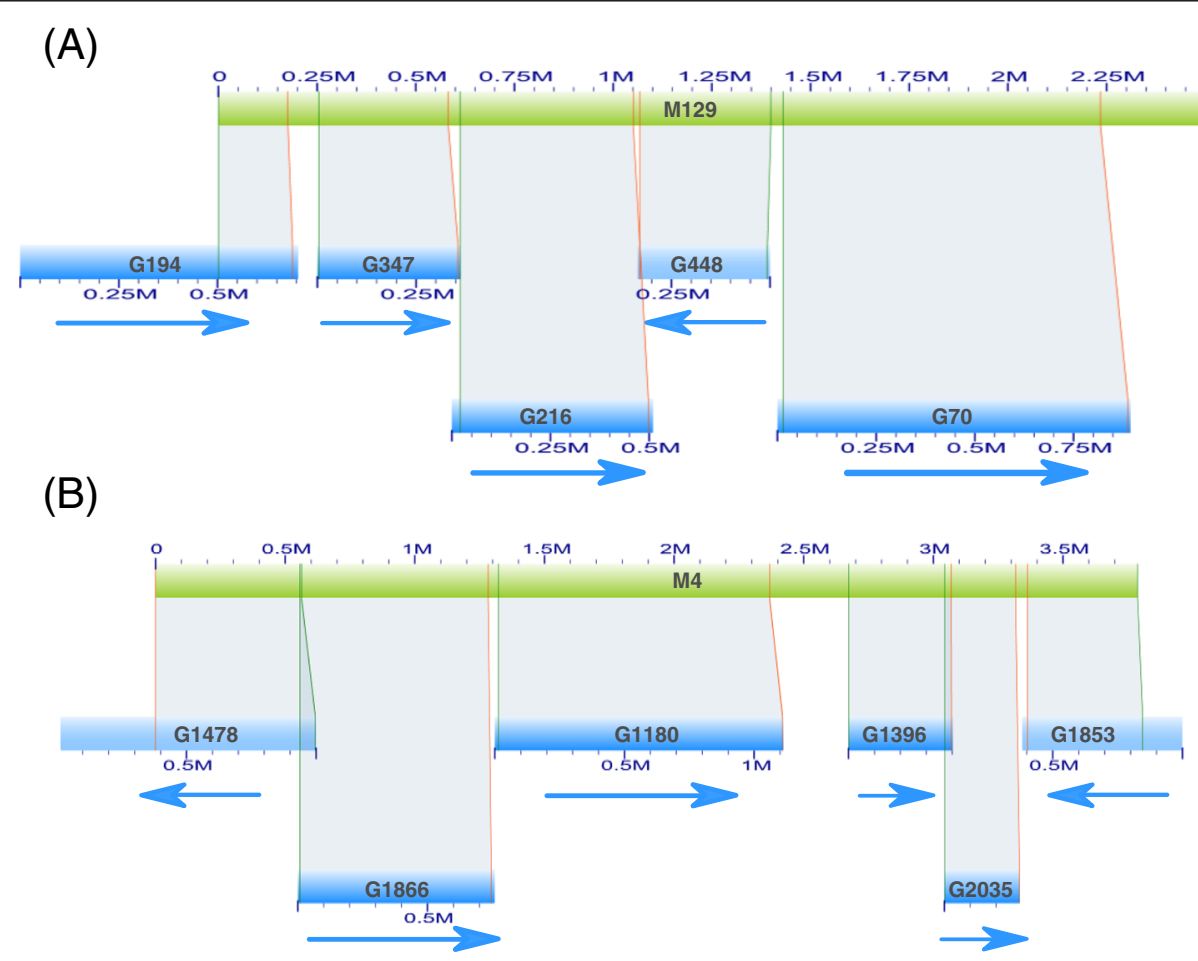

Fig. 6 The validation of genome map by multi-alignments from genome sequences. Two hybrid alignment examples were illuminated for M129 of $2014 \mathrm{draft}$ (a) and M4 of $2015 \mathrm{draft}(\mathbf{b})$. The colour and name scheme in genome map and genome sequences was identical with those in Fig. 5. The gaps between genome sequences may represent un-sampled or low-complexity regions in genomic NGS sequencing. The arrows beneath genome sequences showed the mapping direction along genome maps 
for further experiments: 1 ) the concentration of DNA ranged from $35 n g / \mu L$ to $200 n g / \mu L$; 2) the coefficient of variation $(\mathrm{CV})$ from top, middle, and bottom of the DNA solution was less than $25 \%$; 3) contained mega base size DNA as measured by pulsed field gel electrophoresis (PFGE).

\section{DNA molecules labelling and data collection}

After the extraction and quality control of mega-sized DNA molecules, sequence specific labelling of DNA molecules were performed by Nick, Labelling, Repair and Staining steps according to IrysPrep TM NLRS assay $(900 \mathrm{ng})$ : sequence specificity were provided by the Nickase, Nt.BspQ1; labelling was carried out by a nick translation process in the presence of a fluorophorelabelled nucleotide; the labelled nicks were repaired to restore strand integrity; DNA molecules were stained for the backbone visualization. Automated by the Irys system, stained DNA molecules were loaded into BioNano Genomics nanochannel chips by electrophoresis. Twelve volts were applied to concentrate DNA molecules into the port, thirty volts were used to unwound DNA molecules within the pillar structures and to move long molecules into nanochannels with buffers, and ten volts was applied to linearize DNA molecules in $45 \times 45 \mathrm{~nm}$ nanochannels. Label positions and lengths of DNA molecules were recorded by the on-board CCD camera using green and blue lasers in the BioNano Genomics Irys system.

\section{Data conversion and bioinformatics processes}

Digital images illuminating nick sites in raw DNA molecules from BioNano Genomics Irys system were processed by IrysView package to obtain basic labelling and DNA length information. The signature patterns in DNA molecules were then clustered and assembled by Assembler in IrysView package as described in previous publication [34]. Briefly, DNA molecules were firstly clustered by label distribution with the $\mathrm{R}$ package of fastcluster [41], then label positions along DNA molecules in one cluster were fitted by Gaussian functions. The final assembled maps were constructed by identified peaks in the fitted curves.

For the hybrid alignment of draft genome and genome maps, genome sequences were "digested" into signature patterns according to the restraint site of Nt.BspQ1 by knicker software (http://www.bnxinstall.com/knickers/ Knickers.htm). The alignment with genome maps was performed with RefAligner utility in IrysView package. The parameters for the average false positive (FP) and false negative (FN) were set to 1.5 and 0.15 (-FP 1.5 -FN $0.15)$, respectively. The resulted alignment information, including confidence score [42], was used for the following statistics, such as mapping length ratio calculation (alignment length to the fragment length). Because of the flanking regions before the first nicking site and after the last nicking site, we defined a thorough alignment if mapping length ratio $\geq 0.95$. The visualization of the hybrid alignment between genome maps and sequences were performed and snapshot in IrysView.

\section{Availability of supporting data}

The genome map data of large yellow croaker are accessible on lycgenomics.jmu.edu.cn/pm. Raw DNA molecules cmap files are available in additional files.

\section{Additional file}

Additional file 1: Table S1. DNA molecule statistics with various length filtering. Figure S1. The schematic illustration of the genome map assembly from DNA molecules. (DOCX $429 \mathrm{~kb}$ )

\section{Abbreviations}

AFLP: Amplified fragment length polymorphism; GWAS: Genome-wide association studies; NGS: Next Generation Sequencing; BPP: Bases per pixel; PMBCs: Peripheral mononuclear cells; CV: Coefficient of variation;

PFGE: Pulsed field gel electrophoresis; FP: False positive; FN: False negative.

\section{Competing interests}

The authors declare no competing financial interests. Fengshou $\mathrm{Ma}$ is an employee in Genergy Inc.. This does not alter the authors' adherence to BMC genomics journal policy. The research does not receive any funding from any affiliation to this company.

\section{Authors' contributions}

Z-YW and JL conceived and designed the study; WC conducted sample collection and molecular experiment; SJX and FM performed the bioinformatics analysis; SJX, LF and SBX built the online genome map platform; SJX wrote the manuscript. All authors read and approved the final manuscript.

\section{Acknowledgements}

This work was supported by grants from National '863' Project of China (2012AA10A403), National Natural Science Foundation of China (U1205122) and the Foundation for Innovation Research Team of Jimer University (2010A02).

\section{Author details}

${ }^{1}$ Key Laboratory of Healthy Mariculture in the East China Sea, Ministry of Agriculture; Fisheries College, Jimei University, Yindou Road, Xiamen, P.R. China. ${ }^{2}$ Chinese Academy of Fishery Sciences, Yongding Road, Beijing, P.R. China. ${ }^{3}$ Genergy Inc., Shanghai, P.R. China.

Received: 9 April 2015 Accepted: 21 August 2015

Published online: 03 September 2015

\section{References}

1. Liu M, Mitcheson D, Sadovy Y. Profile of a fishery collapse: why mariculture failed to save the large yellow croaker. Fish Fish. 2008;9(3):219-42.

2. Wang ZY. Progress of Genetic Improvement and the Whole Genome Selection Research Program in Large Yellow Croaker. In: PAG Asia 2013. Plant and Animal Genome.

3. Zhong Y, Power G. Fisheries in China: progress, problems, and prospects. Can J Fish Aquat Sci. 1997;54(1):224-38.

4. Davidson C. Science in contemporary China: Stanford University Press. 1980.

5. Han F, Wang X, Wang Z. Characterization of myosin light chain gene up-regulated in the large yellow croaker immunity by interaction with RanGTPase. Gene. 2013;514(1):54-61.

6. Li HX, Lu XJ, Li CH, Chen J. Molecular characterization and functional analysis of two distinct liver-expressed antimicrobial peptide 2 (LEAP-2) 
genes in large yellow croaker (Larimichthys crocea). Fish Shellfish Immunol. 2014:38(2):330-9.

7. Cui Z, LiU Y, Li CP, You F, Chu KH. The complete mitochondrial genome of the large yellow croaker, Larimichthys crocea (Perciformes, Sciaenidae): unusual features of its control region and the phylogenetic position of the Sciaenidae. Gene. 2009;432(1):33-43.

8. Mu Y, Ding F, Cui P, Ao J, Hu S, Chen X. Transcriptome and expression profiling analysis revealed changes of multiple signaling pathways involved in immunity in the large yellow croaker during Aeromonas hydrophila infection. BMC Genomics. 2010;11(1):506.

9. Ning Y, Liu X, Wang ZY, Guo W, Li Y, Xie F. A genetic map of large yellow croaker Pseudosciaena crocea. Aquaculture. 2007;264(1):16-26.

10. Ye H, Liu Y, Liu X, Wang X, Wang Z. Genetic mapping and QTL analysis of growth traits in the large yellow croaker Larimichthys crocea. Mar Biotechnol. 2014;16(6):729-38.

11. Wu C, Zhang D, Kan M, Lv Z, Zhu A, Su Y, et al. The draft genome of the large yellow croaker reveals well-developed innate immunity. Nat Commun. 2014:5:5227.

12. Ao J, Mu Y, Xiang LX, Fan D, Feng M, Zhang S, et al. Genome sequencing of the perciform fish Larimichthys crocea provides insights into molecular and genetic mechanisms of stress adaptation. PLoS Genet. 2015;11(4), e1005118.

13. Liu Z. Next generation sequencing and whole genome selection in aquaculture: Wiley Online Library. 2011.

14. Yue GH. Recent advances of genome mapping and marker-assisted selection in aquaculture. Fish Fish. 2014;15(3):376-96.

15. Pareek CS, Smoczynski R, Tretyn A. Sequencing technologies and genome sequencing. J Appl Genet. 2011;52(4):413-35

16. Alkan C, Sajjadian S, Eichler EE. Limitations of next-generation genome sequence assembly. Nat Methods. 2011;8(1):61-5.

17. McPherson JD, Marra M, Hillier L, Waterston RH, Chinwalla A, Wallis J, et al. A physical map of the human genome. Nature. 2001;409(6822):934-41.

18. Dong $Y$, Xie M, Jiang Y, Xiao N, Du X, Zhang W, et al. Sequencing and automated whole-genome optical mapping of the genome of a domestic goat (Capra hircus). Nat Biotechnol. 2013;31(2):135-41.

19. Xu P, Wang J, Wang J, Cui R, Li Y, Zhao Z, et al. Generation of the first BAC-based physical map of the common carp genome. BMC Genomics. 2011;12(1):537.

20. Katagiri T, Kidd C, Tomasino E, Davis JT, Wishon C, Stern JE, et al. A BAC-based physical map of the Nile tilapia genome. BMC Genomics. 2005;6(1):89.

21. Ng SH, Artieri CG, Bosdet IE, Chiu R, Danzmann RG, Davidson WS, et al. A physical map of the genome of Atlantic salmon, Salmo salar. Genomics. 2005;86(4):396-404.

22. Xu P, Wang S, Liu L, Thorsen J, Kucuktas H, Liu Z. A BAC-based physical map of the channel catfish genome. Genomics. 2007;90(3):380-8.

23. Xia JH, Feng F, Lin G, Wang CM, Yue GH. A first generation BAC-based physical map of the Asian seabass (Lates calcarifer). PLoS One. 2010;5(8), e11974.

24. Genet C, Dehais P, Palti Y, Gao G, Gavory F, Wincker P, et al. Analysis of BAC-end sequences in rainbow trout: content characterization and assessment of synteny between trout and other fish genomes. BMC Genomics. 2011;12(1):314.

25. Zhang J, Shao C, Zhang L, Liu K, Gao F, Dong Z, et al. A first generation BAC-based physical map of the half-smooth tongue sole (Cynoglossus semilaevis) genome. BMC Genomics. 2014;15(1):215.

26. Samad A, Huff E, Cai W, Schwartz DC. Optical mapping: a novel, singlemolecule approach to genomic analysis. Genome Res. 1995;5(1):1-4.

27. Das SK, Austin MD, Akana MC, Deshpande P, Cao H, Xiao M. Single molecule linear analysis of DNA in nano-channel labeled with sequence specific fluorescent probes. Nucleic Acids Res. 2010;38(18):e177.

28. Reisner W, Larsen NB, Silahtaroglu A, Kristensen A, Tommerup N, Tegenfeldt $\mathrm{JO}$, et al. Single-molecule denaturation mapping of DNA in nanofluidic channels. Proc Natl Acad Sci. 2010;107(30):13294-9.

29. Xiao M, Phong A, Ha C, Chan T-F, Cai D, Leung L, et al. Rapid DNA mapping by fluorescent single molecule detection. Nucleic Acids Res. 2007;35(3):e16.

30. Aston C, Mishra B, Schwartz DC. Optical mapping and its potential for large-scale sequencing projects. Trends Biotechnol. 1999;17(7):297-302.

31. Meng X, Benson K, Chada K, Huff EJ, Schwartz DC. Optical mapping of lambda bacteriophage clones using restriction endonucleases. Nat Genet. 1995;9(4):432-8

32. Lai Z, Jing J, Aston C, Clarke V, Apodaca J, Dimalanta ET, et al. A shotgun optical map of the entire Plasmodium falciparum genome. Nat Genet. 1999;23(3):309-13.

33. Latreille P, Norton S, Goldman BS, Henkhaus J, Miller N, Barbazuk B, et al. Optical mapping as a routine tool for bacterial genome sequence finishing. BMC Genomics. 2007;8(1):321.
34. Lam ET, Hastie A, Lin C, Ehrlich D, Das SK, Austin MD, et al. Genome mapping on nanochannel arrays for structural variation analysis and sequence assembly. Nat Biotechnol. 2012;30(8):771-6.

35. Cao H, Hastie AR, Cao D, Lam ET, Sun Y, Huang H, et al. Rapid detection of structural variation in a human genome using nanochannel-based genome mapping technology. GigaScience. 2014;3(1):34.

36. Hastie AR, Dong L, Smith A, Finklestein J, Lam ET, Huo N, et al. Rapid genome mapping in nanochannel arrays for highly complete and accurate de novo sequence assembly of the complex Aegilops tauschii genome. PLoS One. 2013;8(2), e55864.

37. Majesta O, Searles VB, Dickens CM, Astling D, Albracht D, Mak AC, et al. Sequence and assembly of the DUF1220-rich 1q21 region using a haploid human genome. BMC Genomics. 2014;15(1):387.

38. Zhang X, Zhao C, Huang C, Duan H, Huan P, Liu C, et al. A BAC-based physical map of Zhikong scallop (Chlamys farreri Jones et Preston). PLoS One. 2011;6(11), e27612.

39. Chen Z, Wang Z, Liu X, Jiang Y, Cai M. Area and physical length of metaphase chromosomes in large yellow croaker (Larimichthys crocea) (in chinese). J Fish China. 2014;5:002

40. Lewin HA, Larkin DM, Pontius J, O'Brien SJ. Every genome sequence needs a good map. Genome Res. 2009;19(11):1925-8.

41. Müllner D. fastcluster: fast hierarchical, agglomerative clustering routines for R and Python. J Stat Softw. 2013;53(9):1-18.

42. Anantharaman T, Mishra B. False Positives in Genomic Map Assembly and Sequence Validation. In: Algorithms in Bioinformatics. Springer: Berlin Heidelberg; 2001: 27-40.

\section{Submit your next manuscript to BioMed Central and take full advantage of:}

- Convenient online submission

- Thorough peer review

- No space constraints or color figure charges

- Immediate publication on acceptance

- Inclusion in PubMed, CAS, Scopus and Google Scholar

- Research which is freely available for redistribution 